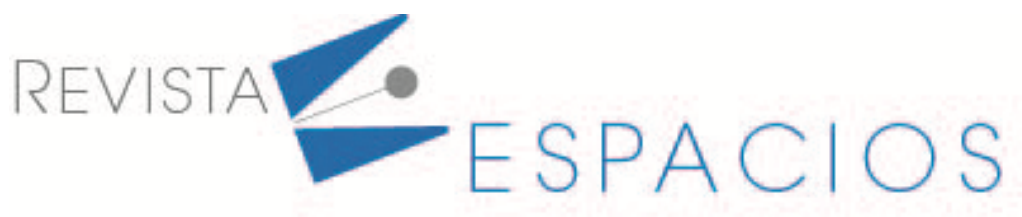

Vol. 41 (45) $2020 \cdot$ Art. 25

Recibido/Received: 21/08/2020 • Aprobado/Approved: 29/09/2020 • Publicado/Published: 26/11/2020

\title{
La seguridad alimentaria: una revisión sistemática con análisis no convencional
}

\section{Food security: a systematic review with unconventional analysis}

\author{
RAMIREZ, Roberth F. ${ }^{1}$ \\ VARGAS, Pablo L. ${ }^{2}$ \\ CARDENAS, Olimpo S. ${ }^{3}$

\section{Resumen} \\ La seguridad alimentaria es un tema en discusión actual; su relación con el hambre, y con otros \\ indicadores de desarrollo humano, ha hecho que su inclusión en la agenda mundial sea de alto interés. \\ Los diferentes abordajes van desde la conceptualización, dimensionamiento, medición, hasta profundas \\ reflexiones. A partir de la revisión sistemática y el análisis de la literatura científica, se identifican áreas \\ emergentes en la temática. El objetivo de esta investigación es sustentar la importancia que tienen los \\ diferentes enfoques para afrontar la seguridad alimentaria como aspecto clave para la sostenibilidad. \\ Se concluye que el uso de cuestionarios para medir la percepción de seguridad/inseguridad alimentaria \\ en el hogar toma vigencia en la actualidad; la escala FIES es muy utilizada por la validez y fiabilidad que \\ ha mostrado en los estudios así como por su facíl de aplicación. \\ Palabras clave: seguridad alimentaria, hambre, sostenibilidad
}

\begin{abstract}
Food security is a current topic of discussion; its relationship with hunger, and with other indicators of human development, has made its inclusion on the global agenda of high interest. The different approaches range from conceptualization, dimensioning, measurement, to deep reflections. The objective of this research is to support the importance of different approaches to address food security as a key aspect for sustainability, through literature review.

key words: food security, hunger, sustainability
\end{abstract}

\section{Introducción}

La seguridad alimentaria (SA) es la situación en la que todas las personas, en todo momento, tienen acceso físico y económico a suficientes alimentos inocuos y nutritivos para satisfacer sus necesidades alimenticias y desarrollar una vida saludable (EC-FAO Food Security, 2008). Garantizar la seguridad alimentaria y nutricional, constituye uno de los elementos transversales y fundamentales para certificar el cumplimiento de los 17 ODS de la Agenda 2030 para el Desarrollo Sostenible (FAO, OPS, WFP y UNICEF, 2018), (FAO, 2018).

\footnotetext{
${ }^{1}$ Economista-MAE-Docente, Facultad de Ciencias Administrativas, Universidad de Guayaquil, roberth.ramirezgr@ug.edu.ec

2 Ingeniero Comercial-MGS-Docente, Facultad de Ciencias Economicas, Universidad de Guayaquil, pablo.vargasram@ug.edu.ec

${ }^{3}$ Ingeniero Comercial-MAE-Docente, Facultad de Ciencias Administrativas, Universidad de Guayaquil, olimpo.cardenasro@ug.edu.ec
} 
La visión transformadora de la mencionada Agenda 2030 para el Desarrollo Sostenible reconoce que el mundo en su cambio exige vivir sin hambre, sin inseguridad alimentaria y sin malnutrición, estos son nuevos desafíos que deben superarse; cambios que repercuten en la manera de producir, distribuir y consumir alimentos a nivel mundial y así poder lograr nuevos desafíos para la seguridad alimentaria, la nutrición y la salud. Esto implica no limitarse a garantizar el acceso a alimentos suficientes, sino también nutritivos que constituyan una dieta saludable para la población (FAO, FIDA, UNICEF, WFP Y OMS, 2019).

En los últimos años la seguridad alimentaria es de los temas más importantes en la agenda económica mundial (Nande, Pérez, Martínez, \& Rangel, 2019); ante las crecientes tendencias negativas, que incluyen el crecimiento de la población mundial, la urbanización progresiva y los cambios climáticos, incrementan preocupaciones sobre la posibilidad de garantizar la seguridad alimentaria a escala mundial (Marzeda-Mlynarska, 2019).

El objetivo de esta investigación es sustentar la importancia que tienen los diferentes enfoques para abordar la seguridad alimentaria. El resto del documento está organizado de la siguiente manera: después de la introducción, se presentan los aspectos pertinentes para la comprensión del alcalce de este documento como lo son seguridad e inseguridad alimentaria, y se discuten trabajos previos en la literatura en esta área de investigación; la siguiente sección, cubre los aspectos metodológicos utilizados en esta revisión sistemática de la literatura; luego se presentan los resultados del análisis de los hallazgos de estudios seleccionados; y finalmente, se presentan algunas implicaciones, y se extraen conclusiones.

\section{Metodología}

El presente estudio sigue un proceso de revisión de la literatura, que incluye pasos: alcance, planificación, identificación/búsqueda, selección, elegibilidad/evaluación, presentación/interpretación. A continuación, una breve descripción de cada uno (Koutsos, Menexes, \& Dordas, 2019)

- Alcance, es la guía la revisión, consta de: desarrollar un protocolo de revisión, identificación de unos pocos estudios relevantes para un estudio piloto de revisión, y una búsqueda previa sobre el tema.

- Planificación, consiste en selecionar las principales palabras clave y cómo se realizará la búsqueda. Es decir el desarrollo de la estrategia de búsqueda y la identificación de bases de datos digitales apropiadas.

- Identificación/busqueda, incluye: implementación de la búsqueda predefinida; revisión de los artículos resultantes; hacer cambios de ser necesario; realizar búsquedas adicionales; buscar fuentes adicionales para identificar artículos, y seleccionar manualmente estudios adicionales.

- Selección, implica la gestión de los artículos resultantes, e involucra la exportación de citas, importar las citas eliminar duplicados, evaluar la información del artículo y un examen exhaustivo de los artículos seleccionados.

- Elegibilidad/evaluación, se recomienda establecer criterios de inclusión y exclusión; definiendo la estrategia para la fuerza de la evidencia; evaluar artículos basados; evaluar los tipos de sesgo que puede existe, y leer en profundidad el texto completo.

- Presentación/interpretación, finaliza en proceso con la sinopsis de los resultados de la revisión sistemática; estudio de la heterogeneidad de los estudios; presentación de los resultados; interpretación de los hallazgos; discusión sobre la generalización de las conclusiones; limitaciones de la revisión sistemática y recomendaciones para más investigación.

Siguiendo las directrices planteadas, la revisión sistemática de literatura se realizó con los siguientes parámetros:

- Luego de la revisión previa de artículos relacionados a SA, durante los meses marzo-abril 2020, se estableció revisar a través del banco de publicaciones Jstor, palabras clave, seguridad alimentaria, medición, food security, measurement, preferiblemente de acceso abierto, publicaciones desde 2016 al 
2020, idioma español o inglés, en Journal, centrados en estudios de la población, ciencias económicas y políticas públicas.

- Resultando 69 artículos, de los que se revisaron 19 exhaustivamente los que mostraban la escala de medición de SA, incluyendo 8 manualmente que aunque fuera de fecha, contienen información oportuna. De la revison se sintetizó la información con énfasis en uso de escalas de medición por ser las de más fácil aplicación. Enseguida se muestra el cuadro 1 con todos los artículos revisados. Cave resaltar, que los artículos seleccionados son los considerados de alta relevancia por presentar la forma de estimación de la SA; así los artículos de relevancia media se consideraron aquellos que no muestran las escalas/instrumentos de medición; y los de baja relevancia aquellos que refieren el tema de manera tangencial pero con poca profundidad.

\section{Resultados}

\subsection{La seguridad alimentaria: una discusión de su definición}

El término de seguridad alimentaria puede resultar confuso desde un punto de vista conceptual (UrquíaFernández, 2014). El concepto de SA ha estado sujeto a varios cambios a través de la historia desde su aparición (Rodríguez Quirós, 2017). Continuar los estudios sobre seguridad alimentaria solamente desde un enfoque teórico o una metodología específica conlleva el riesgo de cosificar el concepto, resultando inevitable la cuantificación y descripción, y contribuir desde la ciencia a mejorar las condiciones de vida de las personas (Pérez Garcés \& Silva Quiroz, 2019).

El concepto de Seguridad Alimentaria Familiar surge a finales de los años ochenta y se ha mantenido dominante desde entonces en los debates teóricos, al ser progresivamente asumido por círculos académicos y por diferentes organismos internacionales (Flores, 2019). La SA es una idea que data del siglo XX (Féliz-Varduzco, AboitesManrique, \& Castro-Lugo, 2018).

En la cumbre mundial de alimentos 1974 se definió la seguridad alimentaria limitándola solo a la disponibilidad de alimentos, concepto que se amplia en 1986 cuando se agrega que se requieren recursos económicos para lograr el acceso a dichos alimentos. Posteriormente se presenta un concepto integral abarcando disponibilidad alimentaria, acceso a los alimentos, estabilidad de los alimentos y utilización biológica (González-León, 2017).

En 1990 se reconoce que la seguridad alimentaria incluye aspectos individuales como los relacionados a la malnutrición proteínico-energética y por ello se amplía el concepto e incorpora el equilibrio nutricional, así también las preferencias alimentarias, sociales y culturales. Con esto se implica un concepto no tan sencillo y se le incorpora el aprovechamiento de los alimentos, posteriormente por aspectos como socioeconómicos, ciclos ambientales, precios, se incorporó la estabilidad, referida a la disponibilidad y acceso a los alimentos (Castell, De la Cruz, Pérez, \& Aranceta, 2015).

A partir del concepto de la World Food Summit (FAO, 1996), la seguridad alimentaria existe cuando todas las personas, en todo momento, tienen acceso físico y económico a suficientes alimentos seguros y nutritivos que satisfagan sus necesidades dietéticas y preferencias alimentarias para una vida activa y saludable; la FAO (2008), identifica cuatro dimensiones, Disponibilidad física de los alimentos, Acceso económico y físico a los alimentos, Utilización de los alimentos y Estabilidad de las otras tres dimensiones a lo largo del tiempo. Para alcanzar los objetivos de SA, las cuatro dimensiones deben cumplirse simultáneamente (EC-FAO Food Security, 2008). A pesar del consenso en torno a esta definición, existen complicaciones en la interpretación del mismo por uso de términos relacionados como inseguridad alimentaria, seguridad alimentaria y nutricional, inseguridad nutricional y hambre, lo que dificulta adoptar de una versión que se ajuste con precisión a una métrica particular (Castell, De la Cruz, Pérez, \& Aranceta, 2015). 


\subsection{La seguridad alimentaria y la inseguridad alimentaria}

El desafío actual en el tema de la Inseguridad Alimentaria (IA) es creciente, las estimaciones son preocupantes e indican que una de cada tres personas se ven afectadas por la desnutrición, es decir, deficiencias de micronutrientes y/o sobrepeso y obesidad. De allí la importancia de reconocer el objetivo número 2 de la agenda 2030 para el desarrollo sostenible "Poner fin al hambre, lograr la seguridad alimentaria y la mejora de la nutrición y promover la agricultura sostenible". Este objetivo equilibra el crecimiento, la sostenibilidad ambiental, reducción de vulnerabilidades, y tendencias adversas como el cambio climático; dado que estos contribuyen a una mayor inseguridad alimentaria (Naciones Unidas, 2018), (FAO, IFAD, UNICEF, WFP y WHO, 2018), (FAO, IFAD, UNICEF, WFP y WHO, 2018).

Es de considerar que la reducción del hambre es un problema que no se corresponde solo a ingresos suficientes, incluye otros aspectos y por ellos debe afrontarse multidimensionalmente. Uno de ellos es lo relacionado a la falta de seguridad alimentaria que se puede traducir en la escasez de alimentos de las personas, en este sentido la existencia de seguridad es el acceso físico y económico de manera constante a alimentos suficientes que garanticen una vida saludable y productiva. Es por ello que el desarrollo, validación y monitoreo de medidas de seguridad alimentaria apropiadas es de gran importancia en las evaluaciones sobre el tema; ejemplos de aspectos a revisar pueden ser la diversidad dietética del hogar, meses de aprovisionamiento adecuado de alimentos en el hogar y escala de acceso de la inseguridad alimentaria en el hogar, que son ampliamente utilizadas en proyectos de desarrollo por su fácil implementación (Lopera, González, Twyman, Useche, \& Talsma, 2018).

A lo largo del tiempo, el concepto de SA ha evolucionado ampliamente, fue necesario un cambio, debido a que inicialmente se basó en la disponibilidad de los alimentos como una estrategia, sin embargo, la experiencia demuestra que la referida disponibilidad es un elemento más en un contexto social y económico más complejo, relacionado con el sistema alimentario de cada país.

De este modo, decir que existe seguridad alimentaria cuando las personas tienen en todo momento acceso a alimentos suficientes, sanos y nutritivos, que se caracteriza por la disponibilidad, el acceso, la estabilidad y el uso no es suficiente. Se ha incluido de manera progresiva el componente de la nutrición que no se relaciona solamente con aspectos médicos, sino también a los servicios de sanidad y al conocimiento imprescindible que deben poseer las personas sobre buenas prácticas para su desarrollo. De allí se desprende la inseguridad alimentaria, un problema multidimensional que se origina en el modo de vida, de alimentación, de la situación económica y social de las personas. Y que está muy lejos de ser un problema de producción agrícola como generalmente se asocia (Brun, 2015).

Sobre los aspectos que contempla la SA se destaca, la disponibilidad al incluir aspectos relacionados a la oferta y se evalúa a nivel nacional, considera la existencia de alimentos de manera oportuna. El acceso se divide en físico y económico, se refiere a cómo las personas adquieren los alimentos que consumen. El económico lo indican los ingresos, los precios de los alimentos, las posibilidades para producir los propios alimentos y la accesibilidad al apoyo social; el acceso físico se asocia con la infraestructura física que permite acceder a los alimentos (GarcíaUrdaneta \& Pérez, 2016).

En este sentido, la SA baja aparece cuando las personas tienen una reducción en la calidad, la variedad, la deseabilidad y hasta en la ingesta de alimentos. Describiendo patrones alimentarios modificados y disminución de la ingesta por falta de recursos. Una muy baja seguridad alimentaria se relaciona con: mayor ingesta de calorías y mayor porcentaje de calorías que provienen de grasa y azúcar añadida; menos consumo de frutas, verduras, carnes, pescado y productos lácteos; mayor índice de masa corporal; salud deficiente y un desarrollo riesgoso en los niños (Salvador Castell, Ngo De la Cruz, Pérez Rodrigo, \& Aranceta, 2015). 
En este orden de ideas, la IA es considerada como la disponibilidad limitada o incierta de alimentos nutricionalmente adecuados e inocuos o la capacidad limitada o incierta de adquirir alimentos adecuados por medios socialmente aceptables (FAO, 1996).

Antes de los 90, la comunidad científica y los sectores encargados del tema, consideraban a la inseguridad alimentaria, el hambre y la desnutrición como dependientes cronológicos, al considerar a la IA como un entorno donde la realidad social y económica permitía el acceso adecuado a los alimentos; si no existía el consumo adecuado, inmediatamente el hambre se presentaba como una consecuencia fisiológica y de ser crónico este consumo inadecuado la consecuencia física subsecuente era la desnutrición. Sin embargo, el conocimiento actual de esta problemática mundial, señala que esta concepción ha cambiado, y la comprensión de la inseguridad alimentaria, el hambre y la desnutrición tiene otros matices al considerar que esta visión se distorsiona por la forma de estimar la prevalencia, causas, consecuencias y las respuestas con políticas apropiadas, adicionalmente la necesidad de las políticas y programas a aplicarse eficazmente a todos los ámbitos complica aún más el reconocimiento previo de la temática. La inseguridad alimentaria y la consecuente desnutrición son expresiones biológicas o de conducta del mundo social, evolucionan diferente en los diversos países, las causas son múltiples, no han sido comprendidas debidamente y están relacionadas al contexto; están ligadas y con origen en variadas y cambiantes condiciones sociales, económicas, ecológicas y políticas. En este sentido se requieren diversas disciplinas científicas para su abordaje, donde la apreciación, análisis y acción se presente considerando que las estrategias para afrontarlas y las respuestas son dependientes del ámbito particular, evaluando, previendo y aprovechando las posibles respuestas relativas a la población. Estas estrategias deben ir unidas a los procesos sociales, políticos e institucionales (Pelletier, Olson, \& Frongillo, 2003). La inseguridad alimentaria influye en la calidad y cantidad de alimentos consumidos (Leung \& Tester, 2019).

El desarrollo de la IA familiar se relaciona con un gradiente que comienza con la incertidumbre y la preocupación sobre el acceso a alimentos, seguido de un ajuste del presupuesto destinado reduciendo la cantidad y variedad de alimentos, continuando con ajustes más severos donde se ven afectadas las cantidades consumidas y se excluyen algunos alimentos, en la última etapa aparece el hambre primero en adultos y luego en los niños (ver figura 1) (Lopera, González, Twyman, Useche, \& Talsma, 2018).

\subsection{Sobre la estimación de la IA}

Con el fin de identificar grupos con mayor vulnerabilidad nutricional, diversas organizaciones se plantean la necesidad de consensuar indicadores para la medición de la inseguridad alimentaria en los hogares, con base científica pero sin dificultad de aplicación.

El establecimiento de las medidas de inseguridad alimentaria bien desarrolladas permitiría la comparabilidad global y son un componente crítico en la el intercambio de conocimiento e información existente dado que se facilitaría la evaluación del cumplimiento de los Objetivos del Desarrollo Sostenible en los sectores público, privado y civil (Pérez-Escamilla, 2017).

Algunos métodos para la medición de IA son: 1) hojas de balance alimentario; 2) presupuesto del hogar y encuestas sobre gastos; 3) entrevistas de ingesta alimentaria individual; 4) evaluación antropométrica y bioquímica; 5) medida de la percepción de seguridad/inseguridad alimentaria en el hogar. Sin embargo los cuatro (4) primeros tienden a ser extensos, costosos y se enfocan más en informar las consecuencias de la IA en lugar de medir la problemática. Es por ello que usar cuestionarios para medir la percepción de seguridad/inseguridad alimentaria en el hogar toma mayor vigencia (Castell, De la Cruz, Pérez, \& Aranceta, 2015).

En este orden de ideas, el cumplimiento del objetivo 2030 ha resaltado la necesidad de una herramienta global a partir de un modelo teórico integrado. Es así como en el 2013, la Organización de las Naciones Unidas para la 
Agricultura y la Alimentación (FAO), reconociendo la existencia de una brecha en la medición de la IS, tomó medidas para desarrollar una nueva herramienta de esta estimación (Saint Ville, Tsun Po, Sen, \& MelgarQuiñonez, 2019).

La referida herramienta es la escala de experiencia de inseguridad alimentaria (FIES, por sus siglas en inglés: Food Insecurity Experience Scale), esta puede mejorar la gobernanza de la SA, pues facilita la planificación, la toma de decisiones y la implementación, al tiempo que garantiza la transparencia en las publicaciones de resultados, y es un punto de referencia para desarrollar políticas y asignar los recursos. Para el desarrollo FIES se ejecutó un proyecto denominado "Voices of the Hungry", el cual involucró directamente a las personas sobre sus experiencias, y así empoderar la IA al darles una "voz". En 2014, la FAO contrató a Gallup, Inc. para recopilar datos de FIES con una encuesta internacional que se realiza anualmente a personas en aproximadamente 150 países. Entre los precursores de FIES se consideran el Módulo de Encuesta de Seguridad Alimentaria de los Hogares de EE.UU y la Escala de Seguridad Alimentaria de América Latina y el Caribe (Pérez-Escamilla, ShamahLevy, \& Candel, 2017).

La FIES es considerada una forma de realizar un seguimiento del hambre (FAO, FIDA, UNICEF, WFP Y OMS, 2019), (Cafiero, Melgar-Quiñonez, Ballard, \& Kepple, 2014). Este método representa un cambio si se compara con métodos tradicionales que evalúan indirectamente la disponibilidad de alimentos, déficits antropométricos y otros signos de malnutrición. FIES mide el acceso de las personas o los hogares a los alimentos, la gravedad de la inseguridad alimentaria (las limitaciones a su capacidad de obtener alimentos suficientes). Este no pretende cuantificar el consumo de alimentos ni proporciona una evaluación cuantitativa de la calidad de la dieta, por lo que no es una medida de desnutrición y no puede usarse para detectar deficiencias nutricionales u obesidad, no es la herramienta adecuada para controlar la desnutrición o evaluar los resultados específicos de nutrición de los programas y políticas de seguridad alimentaria (FAO, s/f). Esta contempla preguntas referidas a la experiencia o comportamiento de las personas en torno a la inseguridad alimentaria, estás reflejan un incremento gradual de los niveles de severidad cuando los recursos para la obtención de alimentos son limitados (Garzón-Orjuela, Merga-Quiñonez, \& Eslava-Schmalbach, 2018), (Ballard, Kepple, \& Cafiero, 2013). Las preguntas, el dominio de la seguridad alimentaria y la severidad se muestran en la tabla 1.

En este orden de ideas, de las investigaciones de Lopera, González, Twyman, Useche y Talsma (2018) y Salvador, De la Cruz, Pérez y Aranceta (2015), en la tabla 2 se muestran las preguntas genéricas relacionadas tomadas de "Household Food Insecurity Access Scale (HFIAS)" y "Food and Nutrition Technical Assistance III Project (FANTA)", el cual es un cuestionario de IA contiene entre 9 y 15 preguntas referidas a un lapso de entre 4 semanas y 3 meses retrospectivamente, y que miden: 1) ansiedad o incertidumbre; 2) calidad insuficiente y 3) cantidad insuficiente. Primero contempla la ocurrencia de IA en las últimas 4 semanas, luego la frecuencia de la situación. $\mathrm{Y}$ algunos autores han complementado con preguntas sobre la ansiedad y la ingesta de alimentos, aspectos que pueden usarse para estimar el hambre en el hogar (Castell, De la Cruz, Pérez, \& Aranceta, 2015), (Cafiero, MelgarQuiñonez, Ballard, \& Kepple, 2014).

Otras investigaciones combinan cuestionarios que se complementan entre sí, Martins en este caso para indagar el efecto de la inseguridad y diversidad alimentaria, la autopercepción de la calidad de la dieta y el sexo sobre el funcionamiento familiar y la salud mental general (Martins, 2018). El cual en primera instancia analiza aspectos sociodemográficos y la obtención del consentimiento de los sujetos en estudio en sus hogares, para posteriormente apoyarse en el cuestionario de salud general (mide la salud mental en general: depresivo, ansioso y bienestar psicológico); recurriendo también a la escala de acceso a la IA de los hogares (HFIAS) excluyendo la pregunta 9, dado que 8 ostenta alta capacidad discriminatoria; así también se usaron el cuestionario de diversidad alimentaria (para contabilizar el número de grupos de alimentos consumidos); el cuestionario de autopercepción de la calidad de la dieta (evalúa calidad de la dieta nutricional, seguridad 
alimentaria y organoléptica); y la sección del cuestionario "McMaster Family Assessment Device" que mide la dimensión referida al funcionamiento general de la familia. El resultado arrojó, entre otros aspectos, que en la muestra objeto de estudio, existe una moderada dificultad para mantener alimentos nutritivos y suficientes en los hogares, así también el temor por no tener suficiente comida en el hogar y el tener que sacrificar grupos alimentarios de preferencia, se constituyeron como las condiciones de inseguridad más marcadas (Martins, 2018), (Martins, 2017), (Coates, Swindale, \& Bilinsky, 2007).

En la misma tendencia, se encuentran investigaciones como la de Flores (2019), en la que se analizó en Managua la percepción de la SA y Nutricional para identificar a los grupos de riesgo y de mayor vulnerabilidad, el cual obtuvo entre sus resultados, que en el periodo estudiado, el $86 \%$ de los hogares presentan IA por falta de acceso a los alimentos en cantidad y calidad adecuada para tener una vida sana. Este estudio se apoyó en la escala ELCSA (ver tabla 3), que consta de 15 preguntas excluyentes, dirigida a indagar sobre una situación diferente y separadas dos secciones ( 8 preguntas aplicables para los hogares con adultos, y 7 preguntas referidas a condiciones que afectan a los menores de 18 años en el hogar).

Por otro parte, otras investigaciones refieren un instrumento para medir el acceso a la alimentación, uno de los indicadores de carencia social en su metodología para la medición multidimensional de la pobreza, el cual se conoce como la escala Latinoamericana y del Caribe sobre Seguridad Alimentaria (ELCSA), que es resultado de varias experiencias con escalas de medición de inseguridad alimentaria en el hogar, en Estados Unidos como Brasil, Colombia, México y otros países de América Latina y el Caribe. Con diversas validaciones en varios países. Esta permite dimensionar la inseguridad alimentaria en leve, moderada y severa. Presenta la situación no solo con números o un elemento estadístico sino también muestra situaciones, preocupaciones y percepciones sobre la falta de alimentos como una vivencia cotidiana dolorosa y desesperante. Para el caso de estudio se usa la herramienta en México, donde el Consejo Nacional de Evaluación de la Política de Desarrollo Social (CONEVAL) adoptó la ELCSA oficialmente, después de varias adaptaciones. CONEVAL mide el indicador de carencia por acceso a la alimentación, mediante los resultados de la ELCSA que describen la situación de Seguridad Alimentaria en los Hogares en tres niveles (leve, moderado, segura). La ELCSA tiene 15 ítems, pero CONEVAL decidió aumentar una pregunta tal como se muestra en la tabla 4. (Carmona, Paredes, \& Pérez, 2017), (ShamahLevya, MUndo-Rosa, Flores-De la Vega, \& Luiselli-Fernández, 2017)

La escala FIES es referencia usada en diversos entornos, dado que la capacidad de los hogares para acceder a alimentos es dimensión clave para medir objetivamente la SA, es usada como medio de comparación entre países. Así (Cafiero, Viviani, \& Nord, 2017), muestran una investigación donde usan en el modelo Rasch con el FIES de ocho ítems para establecer un indicador de monitoreo global de la inseguridad alimentaria (ver tabla 5). El modelo Rasch modelo logístico uniparamétrico conocido como la "Teoría de Respuesta al Ítem", utilizando la máxima verosimilitud incondicional para realizar un ajuste con su sesgo conocido hacia la sobredispersión de los parámetros del ítem. Se analizó información de FAO 2014 al 2016 de 153 países para estimar y comparar tasas de prevalencia de IA, con el procedimiento basado en la mediana de las severidades normalizadas de cada país, los datos mostraron suficiente consistencia para producir medidas confiables de la prevalencia de inseguridad alimentaria en cada país. FIES se enfoca en un método para medir la capacidad de los hogares para acceder a los alimentos similar al de otros basados en la experiencia (como el Módulo de Encuesta de Seguridad Alimentaria Doméstica de los Estados Unidos (HFSSM), la Escala Brasileira de Insegurança Alimentar (EBIA), la Escala Latinoamericana y Caribena de Seguridad Alimentaria (ELCSA), el Escala Mexicana de Seguridad Alimentaria (EMSA) y la Escala de acceso a la inseguridad alimentaria en el hogar (HFIAS) utilizada en Estados Unidos, Brasil, Canadá, México y otros países para monitorear la SA); sin embargo, la innovación está en la posibilidad de calibrar las medidas con el FIES o con cualquiera de estas otras escalas y los umbrales utilizados para la clasificación, contra una escala de referencia estándar, asegurando así la comparabilidad adecuada de las tasas de prevalencia estimadas y la posibilidad de calcular estimaciones consistentes a nivel regional y global. 
De las las investigaciones se desprende que las escalas para estimación de SA/IA que se están usando e nivel global son las relacionadas con FIES (tabla 6 esquematiza los resultados).

\section{Conclusiones}

La IA en los hogares tiene múltiples efectos, primordialmente los relacionados a la malnutrición, enfermedades, riesgo de conflicto social y político, inestabilidad y reducción de la productividad económica. Este es un tema vigente que sigue en estudio en la comunidad científica, con mayor peso a partir de los 17 objetivos de la agenda 2030 para el desarrollo sostenible.

La injusticia social, la inequidad y la falta de garantías para acceder a derechos económicos, sociales, culturales y medioambientales así como el derecho a los alimentos de la población, son las causas principales de inseguridad alimentaria. Por otro lado, a pesar de la diversidad en la población mundial, la inseguridad alimentaria se asoció comúnmente con determinantes y consecuencias de la seguridad alimentaria como lo son: el ingreso nacional, la salud y el bienestar.

Diversos organismos en varios países, se han propuesto alcanzar un consenso y desarrollar indicadores de medida de inseguridad alimentaria en los hogares. Un método fácil, basado en la experiencia, con validez y fiabilidad, que sea capaz de medir el grado de severidad de la IA. Esto con el fin primordial de detectar grupos más vulnerables e implementar estrategias verdaderamente dirigidas a esta población con problemas.

FIES es una herramienta ampliamente usada y adaptada, se han recolectado con ella datos en más de 150 países en todo el mundo. En su aplicación, un aspecto que resalta es que está regularmente asociada con la incapacidad para acceder a los alimentos por falta de dinero o recursos, resaltando la pobreza como una de las causas. Esta, se está convirtiendo en una escala de referencia global que puede usarse para asegurar estimaciones comparables de prevalencia de inseguridad alimentaria en diferentes poblaciones. En las investigaciones, se evidencia que los formuladores de políticas y académicos reconocen a FIES como la única medida de inseguridad alimentaria a nivel doméstico o individual que brinda la oportunidad de generar medidas estándar de inseguridad alimentaria internacionalmente comparables con detalles sobre los niveles de gravedad. Así, también el método se puede aplicar a otras escalas de seguridad alimentaria ofreciendo la posibilidad de utilizar los datos recopilados con esos instrumentos para producir medidas comparables internacionalmente de la prevalencia de IA. Se espera una adopción más amplia de la FIES o escalas de seguridad alimentaria, dado la garantía de estimaciones comparables de la prevalencia de la inseguridad alimentaria en diferentes poblaciones, la posibilidad de calibrar medidas contra el estándar global, y además de la posibilidad de adicionar preguntas que contribuyan de manera efectiva en la medición de rasgos subyacentes en países o culturas específicas.

Los diferentes medios de estimación de la seguridad alimentaria reflejan bajo complimiento de los objetivos de la Agenda 2030 para el desarrollo sostenible, especifícamente los relacionados a "cero hambre". Como criterio de seguimiento, se evidencia la necesidad de realizar un mayor énfasis en estas mediciones.

La ELCSA es una herramienta de bajo costo, rápida aplicación y fácil interpretación; que ha demostrado una alta validez y confiabilidad en los diversos campos de aplicación. Esta escala tiene gran potencial para seguir contribuyendo a la mejor comprensión de la distribución, causas y consecuencias de la Inseguridad Alimentaria.

\section{Referencias bibliográficas}

Ballard, T., Kepple, A., \& Cafiero, C. (2013). The Food Insecurity Experience Scale. Development of a Global Standard for Monitoring Hunger Worldwide. (FAO, Ed.) Technical Paper. Rome, FAO. 
Brun, M. (2015). Inseguridad alimentaria y nutricional: un desafío importante para el mundo árabe. Afkar Ideas. revista Trimestral para el diálogo entre el Magred.

Cafiero, C., Melgar-Quiñonez, H., Ballard, T., \& Kepple, A. (2014). Validity and reliability of food security measures. Ann N Y Acad Sci2014, 230-248.

Castell, G., De la Cruz, J., Pérez, C., \& Aranceta, J. (2015). Escalas de evaluación de la inseguridad alimentaria en el hogar. Revista Española de Nutrición, 21(1), 270-276.

EC-FAO Food Security. (2008)a. Organización de las Naciones Unidas para la Alimentación y la Agricultura. (E.-F. f. Programme, Ed.) Recuperado el 10 de mayo de 2020, de FAO: http://www.fao.org/3/a-al936e.pdf

EC-FAO Food Security. (2008)b. Organización de las Naciones Unidas para la Alimentación y la Agricultura. (E.F. f. Programme, Ed.) Recuperado el 10 de mayo de 2020, de An Introduction to the Basic Concepts of Food Security: http://www.fao.org/3/a-al936e.pdf

FAO. (1996). Informe de la Cumbre Mundial sobre la Alimentación. ORGANIZACIÓN DE LAS NACIONES UNIDAS

FAO. (s/f). The Food Insecurity Experience Scale. Frequently Asked Questions - FAQs. Food and Agriculture Organization of United Nations. FAO. Obtenido de http://www.fao.org/3/a-bl354e.pdf

FAO, FIDA, UNICEF, WFP Y OMS. (2019). El estado de la Seguridad Alimentaria y la Nutrición en el mundo. Versión Resumida. FAO. FAO. Recuperado el 9 de mayo de 2020, de http://www.fao.org/3/ca5249es/ca5249es.pdf

FAO, IFAD, UNICEF, WFP y WHO. (2018). The State of Food Security and Nutrition in the World 2017. Building resilience for peace and food security. Roma: FAO.

FAO, OPS, WFP y UNICEF. (2018). Panorama de la seguridad alimentaria y nutricional en América Latina y el Caribe 2018. Santiago: FAO.

Féliz-Varduzco, G., Aboites-Manrique, G., \& Castro-Lugo, D. (2018). La seguridad alimentaria y su relación con la suficiencia e incertidumbre del ingreso: un análisis de las percepciones del hogar. Acta Universitaria, 28(4), 74-86.

Flores, C. (2019). Percepción de la Seguridad Alimentaria de los hogares del municipio San Francisco Libre, Managua 2014-2016. Torreón Universitario, 8(22), 6-16.

García-Urdaneta, A., \& Pérez, J. (2016). Marco Conceptual de la Medición de Seguridad Alimentaria (SA): Análisis Comparativo y Crítico De Algunas Métricas. Agroalimentaria, 22(43).

Garzón-Orjuela, N., Merga-Quiñonez, H., \& Eslava-Schmalbach, J. (2018). Escala Basada en la Experiencia de Inseguridad Alimentaria (FIES). Salud Pública de México, 60(5), 510-519.

González-León, C. (2017). Principales fundamentos teórico-metodológicos acerca de la seguridad alimentaria y nutricional y las políticas públicas. (U. C. Villas, Ed.) Convención Colectiva Internaciola. Ciencia, Tecnología y Sociedad, Perspectivas y Retos, 1-16.

Koutsos, T., Menexes, G., \& Dordas, C. (2019). An efficient framework for conducting systematic literature reviews in. Science of the Total Environment, 682, 106-117.

Leung, C., \& Tester, J. (2019). The Association between Food Insecurity and Diet Quality Varies by Race/Ethnicity: An Analysis of National Health and Nutrition Examination Survey 2011-2014 Results. Journal of the Academy of Nutrition and Dietetics, 119(10), 1676-1686. 
Lopera, D., González, C., Twyman, J., Useche, P., \& Talsma, E. (2018). His and Hers, time and income: How intrahousehold dynamics impact nutrition in agricultural households. Harvard Dataverse, 2.

Marzeda-Mlynarska, K. (2019). . "La gobernanza de la seguridad alimentaria en América Latina: desde la cooperación Norte-Sur hacia la cooperación Sur-Sur. Anuario Latinoamericano Ciencia Política y Ralaciones Internacionales, 7(1), 131-151.

Naciones Unidas. (2018). La Agenda 2030 y los Objetivos de Desarrollo Sostenible: una oportunidad para América Latina y el Caribe (LC/G.2681-P/Rev.3).

Nande, E., Pérez, O., Martínez, S., \& Rangel, J. (2019). Seguridad alimentaria y obesidad: un análisis comparativo entre China-México-EU. Portes, Revista Mexicana de Estudios sobre la Cuenca del Pacífico, 13(25), 105-137.

Pelletier, D., Olson, C., \& Frongillo, E. (2003). Inseguridad alimentaria, hambre y desnutrición. En B. Bowman, \& R. Russell, Conocimientos Actuales sobre Nutrición (Vol. 8va. edición, págs. 762-775). Washinton, D.C.: OPS.

Pérez Garcés, R., \& Silva Quiroz, Y. (2019). Enfoques y factores asociados a la inseguridad alimentaria. Revista Salud Pública y NUtrición, 18(1), 15-24.

Pérez-Escamilla, R. (2017). Food security and the 2015-2030 sustainable development goals: from human to planetary health: perspectives and opinions. Current Developments in Nutrition, 1(7), 1-8.

Pérez-Escamilla, R., Shamah-Levy, T., \& Candel, J. (2017). Food security governance in Latin America: principles and the way forward. Global Food Security, 14, 68-72.

Rodríguez Quirós, R. (2017). Seguridad Alimentaria: Evolución conceptual y relación con el cambio climático. Revista Universidad en Diálogo, 7(2), 97-105.

Saint Ville, A., Tsun Po, J., Sen, A., \& Melgar-Quiñonez, H. (2019). Food security and the Food Insecurity Experience Scale (FIES): ensuring progress by 2030. Food Security, 11, 483-491.

Salvador Castell, G., Ngo De la Cruz, J., Pérez Rodrigo, C., \& Aranceta, J. (2015). Revista Española de Nutrición Comunitaria, 21(1), 270-276.

Urquía-Fernández, N. (2014). La Seguridad Alimentaria en México. Salud Pública de México, 56(1), 92-98.

Esta obra está bajo una Licencia Creative Commons Attribución-NoCommercial 4.0 International

\section{(cc) EY-NC}

